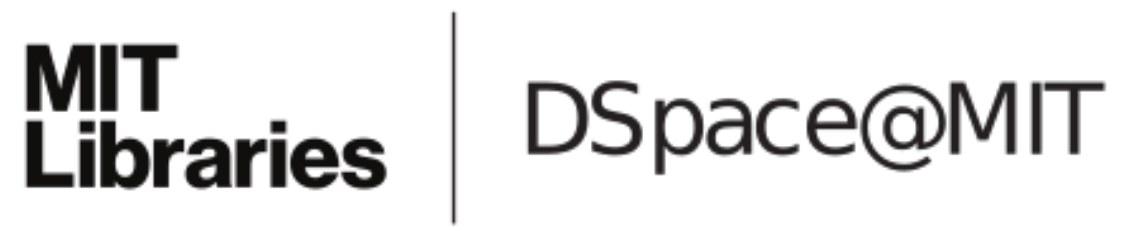

\author{
MIT Open Access Articles
}

A new error bound for reduced basis approximation
of parabolic partial differential equations

The MIT Faculty has made this article openly available. Please share how this access benefits you. Your story matters.

Citation: Urban, Karsten, and Anthony T. Patera. "A New Error Bound for Reduced Basis Approximation of Parabolic Partial Differential Equations." Comptes Rendus Mathematique 350, no. 3-4 (February 2012): 203-207.

As Published: http://dx.doi.org/10.1016/j.crma.2012.01.026

Publisher: Elsevier

Persistent URL: http://hdl.handle.net/1721.1/99384

Version: Author's final manuscript: final author's manuscript post peer review, without publisher's formatting or copy editing

Terms of use: Creative Commons Attribution-Noncommercial-NoDerivatives 


\title{
A New Error Bound for Reduced Basis Approximation of Parabolic Partial Differential Equations
}

\author{
Karsten Urban \\ University of Ulm, Institute for Numerical Mathematics, Helmholtzstr. 18, \\ 89081 Ulm (Germany), karsten.urban@uni-ulm.de \\ Anthony T. Patera \\ Mechanical Engineering Department, Massachusetts Institute of Technology, \\ 77 Massachusetts Ave., Cambridge, MA 02139-4307 (USA), patera@mit.edu
}

\begin{abstract}
We consider a space-time variational formulation for linear parabolic partial differential equations. We introduce an associated Petrov-Galerkin truth finite element discretization with favorable discrete inf-sup constant $\beta_{\delta}: \beta_{\delta}$ is unity for the heat equation; $\beta_{\delta}$ grows only linearly in time for non-coercive (but asymptotically stable) convection operators. The latter in turn permits effective long-time a posteriori error bounds for reduced basis approximations, in sharp contrast to classical (pessimistic) exponentially growing energy estimates.

\section{Résumé}

Nous considérons une formulation variationnelle espace-temps pour les équations différentielles paraboliques linéaires. Nous y associons une discrétisation par éléments finis de Petrov-Galerkin pour laquelle la constante de stabilité inf-sup $\beta_{\delta}$ possède des propriétés agréables : $\beta_{\delta}$ est unité pour l'équation de la chaleur; $\beta_{\delta}$ a une croissance seulement linéaire en temps pour des opérateurs de convection non-coercifs (mais asymptotiquement stables). Dans le cadre des approximations par bases réduites, cette dernière propriété permet d'obtenir des bornes efficaces pour l'erreur a posteriori en temps long, en net contraste avec les estimateurs d'erreur en énergie classiques (pessimistes) qui présentent une croissance exponentielle.
\end{abstract}

Key words: parabolic equations, space-time formulation, inf-sup stability, reduced basis, a posteriori estimation 1991 MSC: 35K15, 65M15, 65M60

\section{Version française abrégée}

Soit l'équation aux dérivées partielles parabolique (1) et sa formulation variationnelle espace-temps : $u \in \mathcal{X}:=L_{2}(I \equiv(0, T] ; V) \cap H_{(0)}^{1}\left(I ; V^{\prime}\right), H_{(0)}^{1}\left(I ; V^{\prime}\right):=\left\{v \in H^{1}\left(I ; V^{\prime}\right): v(0)=0\right\}$, vérifie $(2)$ pour l'espace des fonctions test $\mathcal{Y}:=L_{2}(I ; V)$; ici $b(w, v):=\int_{I}\left[\langle\dot{w}(t), v(t)\rangle_{V^{\prime} \times V}+a(w(t), v(t))\right] d t$ et $f(v):=$ 
$\int_{I}\langle g(t), v(t)\rangle_{V^{\prime} \times V} d t$. Les normes sur $\mathcal{X}$ et $\mathcal{Y}$ sont définies par $\|w\|_{\mathcal{X}}^{2}:=\|w\|_{L_{2}(I ; V)}^{2}+\|\dot{w}\|_{L_{2}\left(I ; V^{\prime}\right)}^{2}+\|w(T)\|_{H}^{2}$ et $\|v\|_{\mathcal{Y}}:=\|v\|_{L_{2}(I ; V)}$. On considère $V=H_{0}^{1}(\Omega)$ (opérateurs spatiaux de $2 \mathrm{e}$ ordre), où $\Omega$ est le domaine spatial. On montre (Proposition 1 basée sur [6]) que pour des problèmes coercifs, la constante inf-sup $\beta$ de $b$ est positive et bornée inférieurement.

Nous introduisons ensuite une discrétisation par éléments finis de Petrov-Galerkin : soit $u_{\delta} \in \mathcal{X}_{\delta}$ vérifiant $b\left(u_{\delta}, v_{\delta}\right)=f\left(v_{\delta}\right), \forall v_{\delta} \in \mathcal{Y}_{\delta}$; avec $\mathcal{X}_{\delta}:=S_{\Delta t} \otimes V_{h}$ et $\mathcal{Y}_{\delta}=Q_{\Delta t} \otimes V_{h}, \delta=(\Delta t, h)$, où $S_{\Delta t}, V_{h}$ et $Q_{\Delta t}$ sont respectivement des éléments finis linéaire par morceaux en temps (de pas $\Delta t$ ) et en espace (de diamètre $h$ ), et constants en temps par morceaux. Cette méthode coïncide avec une discrétisation de Crank-Nicolson en temps, ainsi nos résultats s'appliquent directement à ce schéma standard. Nous définissons une norme modifiée sur $\mathcal{X},\|\| \cdot\|\|_{\mathcal{X}, \delta}$, où la partie $\int_{I}\|w\|_{V}^{2} d t$ de $\|\cdot\|_{\mathcal{X}}$ est remplacée par une somme des moyennes de $w$ sur chaque élément temporel. Nous montrons (Proposition 3) que pour l'équation de la chaleur, les constantes inf-sup discrète $\beta_{\delta}$ et continuité discrète $\gamma_{\delta}$ sont unités.

Nous considérons maintenant une approximation par bases réduites $u_{N}$, comme dans [2]. Nous pouvons construire à la Proposition 5 des bornes sur l'erreur a posteriori pour le champ solution $u_{N}$ et pour la sortie scalaire $s_{N}$. L'utilité de ces bornes est très liée à la dépendance de $\beta_{\delta}$ aux paramètres du problème et au temps final $T$. La procédure de calcul de ces bornes est similaire à la décomposition hors ligne-en ligne des bases réduites standard.

Enfin, nous présentons des résultats numériques pour la constante inf-sup discrète $\beta_{\delta}$. Pour des opérateurs non-coercifs mais asymptotiquement stables (e.g. convection), on observe que le paramètre inf-sup decroît seulement en $\left(\mu_{1} T\right)^{-1}$, où $\mu_{1}$ est la vitesse de déplacement et $T$ le temps final. L'exemple est un domaine spatial unidimensionnel $(0,1)$ avec l'opérateur $\mu_{1}(x-1 / 2) u_{x}-u_{x x}$ pour $\mu_{1}>2 \pi^{2}\left(\mu_{1}<2 \pi^{2}\right.$ entraîne un problème coercif). Les estimateurs associés sont beaucoup plus précis que ceux classiques en énergie qui prédisent une croissance exponentielle en $e^{\mu_{1} T}$ et qui sont donc inutilisables en pratique.

\section{Space-time formulation}

We first formulate a general linear parabolic equation. Consider Hilbert spaces $V \hookrightarrow H \hookrightarrow V^{\prime}$ and an operator $A \in \mathcal{L}\left(V, V^{\prime}\right),\langle A \phi, \psi\rangle_{V^{\prime} \times V}=a(\phi, \psi)$ for $\phi, \psi \in V$. Setting $I:=(0, T], T>0$ and given $g \in L_{2}\left(I ; V^{\prime}\right)$, we look for $u$ such that

$$
\dot{u}(t)+A u(t)=g(t) \text { in } V^{\prime}, t \in I ; \quad u(0)=0,
$$

and an associated output of the form $s:=\int_{I} \ell(u(t)) d t$ for some $\ell \in V^{\prime}$. We restrict ourselves to LTI systems even though some of our results can be extended to a more general situation.

For the derivation of the space-time variational form of (1), we introduce the trial space $\mathcal{X}:=$ $L_{2}(I ; V) \cap H_{(0)}^{1}\left(I ; V^{\prime}\right)$, where $H_{(0)}^{1}\left(I ; V^{\prime}\right):=\left\{v \in H^{1}\left(I ; V^{\prime}\right): v(0)=0\right\}$ with norm $\|w\|_{\mathcal{X}}^{2}:=\|w\|_{L_{2}(I ; V)}^{2}+$ $\|\dot{w}\|_{L_{2}\left(I ; V^{\prime}\right)}^{2}+\|w(T)\|_{H}^{2}$ and the test space $\mathcal{Y}:=L_{2}(I ; V)$ with norm $\|v\|_{\mathcal{Y}}:=\|v\|_{L_{2}(I ; V)}$. Note that $\mathcal{X}=\left(L_{2}(I) \otimes V\right) \cap\left(H_{(0)}^{1}(I) \otimes V^{\prime}\right)$ and $\mathcal{Y}=L_{2}(I) \otimes V$ which will allow for a tensor product discretization. Then definitions $b(w, v):=\int_{I}\left[\langle\dot{w}(t), v(t)\rangle_{V^{\prime} \times V}+a(w(t), v(t))\right] d t$ and $f(v):=\int_{I}\langle g(t), v(t)\rangle_{V^{\prime} \times V} d t$ yield the space-time variational formulation

$$
u \in \mathcal{X}: \quad b(u, v)=f(v), \quad \forall v \in \mathcal{Y} .
$$

The well-posedness of (2) has been shown (under suitable assumptions) in [6, Theorem 5.1].

The approach of [6] can also yield an estimate for the inf-sup constant $\beta:=\inf _{w \in \mathcal{X}} \sup _{v \in \mathcal{Y}} \frac{b(w, v)}{\|w\|_{\mathcal{X}}\|v\|_{\mathcal{Y}}}$. We define $\varrho:=\sup _{0 \neq \phi \in V} \frac{\|\phi\|_{H}}{\|\phi\|_{V}}$ and $\beta_{a}^{*}:=\inf _{\phi \in V} \sup _{\psi \in V} \frac{a(\psi, \phi)}{\|\phi\|_{V}\|\psi\|_{V}}$; we then claim 
Proposition 1 Assume that there exist $M_{a}<\infty, \alpha>0$, and $\lambda \geq 0$ such that $|a(\psi, \phi)| \leq M_{a}\|\psi\|_{V}\|\phi\|_{V}$ (continuity) and $a(\phi, \phi)+\lambda\|\phi\|_{H}^{2} \geq \alpha\|\phi\|_{V}^{2}$ (Gärding) for $v, w \in V$. Then, we obtain the inf-sup lower bound $\beta \geq \beta^{L B}:=\frac{\min \left\{1,\left(\alpha-\lambda \varrho^{2}\right) \min \left\{1, M_{a}^{-2}\right\}\right\}}{\max \left\{1,\left(\beta_{a}^{*}\right)^{-1}\right\} \sqrt{2}}$.

Proof. (Sketch) Let $0 \neq w \in \mathcal{X}$. Set $z_{w}:=\left(A^{*}\right)^{-1} \dot{w}$ (where $A^{*}: V \rightarrow V^{\prime}$ denotes the adjoint of $V$ ) and set $v_{w}:=z_{w}+w \in \mathcal{Y}$. Then, $\left\|v_{w}\right\|_{L_{2}(I ; V)}^{2} \leq 2 \max \left\{1,\left(\beta_{a}^{*}\right)^{-2}\right\}\|w\|_{\mathcal{X}}^{2}$. Using the estimates $\|\dot{w}(t)\|_{V^{\prime}} \leq$ $M_{a}\left\|z_{w}(t)\right\|_{V},\left\langle\dot{w}(t), z_{w}(t)\right\rangle_{V^{\prime} \times V} \geq\left(\alpha-\lambda \varrho^{2}\right) M_{a}^{-2}\|\dot{w}(t)\|_{V^{\prime}}^{2}$ and $a\left(w(t), z_{w}(t)\right)=\frac{1}{2} \frac{d}{d t}\|w(t)\|_{H}^{2}$ we arrive at $b\left(w, v_{w}\right) \geq\left(\alpha-\lambda \varrho^{2}\right)\left(M_{a}^{-2}\|\dot{w}\|_{L_{2}\left(I ; V^{\prime}\right)}^{2}+\|w\|_{L_{2}(I ; V)}^{2}\right)+\|w(T)\|_{H}^{2} \geq \min \left\{\left(\alpha-\lambda \varrho^{2}\right) \min \left\{1, M_{a}^{-2}\right\}, 1\right\}\|w\|_{\mathcal{X}}^{2} \geq$ $\beta^{\mathrm{LB}}\|w\|_{\mathcal{X}}\left\|v_{w}\right\|_{\mathcal{Y}}$, which proves the claim.

Remark 2 Note that $\beta^{L B}$ does not depend on the final time. However, the estimate is only meaningful if $\alpha \geq \lambda \varrho^{2}$, i.e., if the system is coercive. In the non-coercive case, (1) is often transformed via $\hat{u}(t):=$ $e^{-\lambda t} u(t)$ to obtain a coercive problem; however, this yields an inf-sup bound that behaves as $e^{-\lambda T}-$ often extremely pessimistic and clearly unsuitable for error estimation in long-time integration.

\section{Petrov-Galerkin Truth Approximation}

Let $\mathcal{X}_{\delta} \subset \mathcal{X}, \mathcal{Y}_{\delta} \subset \mathcal{Y}$ be finite dimensional subspaces and $u_{\delta} \in \mathcal{X}_{\delta}$ the discrete approximation of (2), i.e., $b\left(u_{\delta}, v_{\delta}\right)=f\left(v_{\delta}\right), \forall v_{\delta} \in \mathcal{Y}_{\delta}, s_{\delta}=\int_{0}^{T} \ell\left(u_{\delta}(t)\right) d t$. Henceforth, we concentrate on the case $H=L_{2}(\Omega)$, $V=H_{0}^{1}(\Omega)$. Let $\mathcal{X}_{\delta}=S_{\Delta t} \otimes V_{h}, \mathcal{Y}_{\delta}=Q_{\Delta t} \otimes V_{h}, \delta=(\Delta t, h)$, where $S_{\Delta t}, V_{h}$ are piecewise linear and $Q_{\Delta t}$ piecewise constant finite elements with respect to triangulations $\mathcal{T}_{h}^{\text {space }}$ in space and $\mathcal{T}_{\Delta t}^{\text {time }} \equiv\left\{t^{i-1} \equiv\right.$ $\left.(i-1) \Delta t<t \leq i \Delta t \equiv t^{i}, 1 \leq i \leq r\right\}$ in time for $\Delta t:=T / r$. This coincides with the Crank-Nicolson (CN) scheme if a trapezoidal approximation of the right-hand side temporal integration is used; hence, we can derive error bounds for the $\mathrm{CN}$ scheme via our space-time formulation.

We introduce a different norm on $\mathcal{X}$ : For $w \in \mathcal{X}$ and $I^{i}:=\left(t^{i-1}, t^{i}\right)$, set $\bar{w}^{i}:=(\Delta t)^{-1} \int_{I^{i}} w(t) d t \in V$ and $\bar{w}:=\sum_{i=1}^{r} \chi_{I^{i}} \otimes \bar{w}^{i} \in L_{2}(I ; V)$, where $\chi_{I^{i}}$ is the characteristic function on $I^{i}$; then, set $\||w|\|_{\mathcal{X}, \delta}^{2}:=$ $\|\dot{w}\|_{L_{2}\left(I ; V^{\prime}\right)}^{2}+\|\bar{w}\|_{L_{2}(I ; V)}^{2}+\|w(T)\|_{H}^{2}$ and the inf-sup parameter $\beta_{\delta}:=\inf _{w_{\delta} \in \mathcal{X}_{\delta}} \sup _{v_{\delta} \in \mathcal{Y}_{\delta}} \frac{b\left(w_{\delta}, v_{\delta}\right)}{\left\|\mid w_{\delta}\right\| \mathcal{X}_{, \delta}\left\|v_{\delta}\right\| \mathcal{Y}}$ as well as the stability parameter $\gamma_{\delta}:=\sup _{w_{\delta} \in \mathcal{X}_{\delta}} \sup _{v_{\delta} \in \mathcal{Y}_{\delta}} \frac{b\left(w_{\delta}, v_{\delta}\right)}{\left\|w_{\delta}\right\| \mathcal{X}_{, \delta}\left\|v_{\delta}\right\|_{\mathcal{Y}}}$.

Proposition 3 Let $a(\cdot, \cdot)$ be symmetric, bounded and coercive and set $\|\phi\|_{V}^{2}:=a(\phi, \phi), \phi \in V$; then we have $\beta_{\delta}=\gamma_{\delta}=1$.

Proof. (Sketch) Since $v_{\delta} \in \mathcal{Y}_{\delta}$ is piecewise constant in time, we have $\int_{I} a\left(w_{\delta}, v_{\delta}\right) d t=\int_{I} a\left(\bar{w}_{\delta}, v_{\delta}\right) d t$ for all $w_{\delta} \in \mathcal{X}_{\delta}$. Hence, $b\left(w_{\delta}, v_{\delta}\right)=\int_{I} a\left(A_{h}^{-1} \dot{w}_{\delta}+\bar{w}_{\delta}, v_{\delta}\right) d t$, where $z_{\delta}:=A_{h}^{-1} \dot{w}_{\delta}$ is defined by $a\left(z_{\delta}, \phi_{h}\right)=$ $\left\langle\dot{w}_{\delta}, \phi_{h}\right\rangle_{V^{\prime} \times V}, \forall \phi_{h} \in V_{h}$. Note that $\left\|A_{h}^{-1} \dot{w}_{\delta}\right\|_{V}=\left\|\dot{w}_{\delta}\right\|_{V^{\prime}}$. We may prove that for any $v_{\delta} \in \mathcal{Y}_{\delta}$ there exists a unique $z_{\delta} \in \mathcal{X}_{\delta}$ such that $\int_{I} a\left(A_{h}^{-1} \dot{z}_{\delta}+\bar{z}_{\delta}, q_{\delta}\right) d t=\int_{I} a\left(v_{\delta}, q_{\delta}\right) d t$ for all $q_{\delta} \in \mathcal{Y}_{\delta}$. Note that $v_{\delta}:=A_{h}^{-1} \dot{z}_{\delta}+\bar{z}_{\delta} \in \mathcal{Y}_{\delta}$ for $z_{\delta} \in \mathcal{X}_{\delta}$ and we obtain $b\left(w_{\delta}, v_{\delta}\right)=\int_{I} a\left(A_{h}^{-1} \dot{w}_{\delta}+\bar{w}_{\delta}, A_{h}^{-1} \dot{z}_{\delta}+\bar{z}_{\delta}\right) d t$ so that $\sup _{v_{\delta} \in \mathcal{Y}_{\delta}} \frac{b\left(w_{\delta}, v_{\delta}\right)}{\left\|v_{\delta}\right\| \mathcal{Y}}=\sup _{v_{\delta} \in \mathcal{Y}_{\delta}}\left(\int_{I} a\left(A_{h}^{-1} \dot{w}_{\delta}+\bar{w}_{\delta}, A_{h}^{-1} \dot{z}_{\delta}+\bar{z}_{\delta}\right) d t\right) /\left\|A_{h}^{-1} \dot{z}_{\delta}+\bar{z}_{\delta}\right\|_{\mathcal{Y}}=\left\|A_{h}^{-1} \dot{w}_{\delta}+\bar{w}_{\delta}\right\|_{\mathcal{Y}}$. The fact that $\left\|A_{h}^{-1} \dot{w}_{\delta}+\bar{w}_{\delta}\right\|_{\mathcal{Y}}^{2}=\left\|A_{h}^{-1} \dot{w}_{\delta}\right\|_{L_{2}(I ; V)}^{2}+\left\|\bar{w}_{\delta}\right\|_{L_{2}(I ; V)}^{2}+2 \int_{I}\left\langle\dot{w}_{\delta}, \bar{w}_{\delta}\right\rangle_{V^{\prime} \times V} d t=\left\|\dot{w}_{\delta}\right\|_{L_{2}\left(I ; V^{\prime}\right)}^{2}+\left\|\bar{w}_{\delta}\right\|_{L_{2}(I ; V)}^{2}+$ $\left\|w_{\delta}(T)\right\|_{H}^{2}=\left\||| w_{\delta} \mid\right\|_{\mathcal{X}, \delta}^{2}$ proves the assertion.

Remark 4 Proposition 3 also shows the well-posedness of the discrete problem with continuity and infsup constant being unity. 


\section{The Reduced Basis Method (RBM)}

Now, let $\mu=\left(\mu_{1}, \mu_{2}\right) \in \mathcal{D}:=\mathbb{R}^{2}$ be a parameter vector and $A=A(\mu):=-\Delta u+\mu_{1} \boldsymbol{\beta}(x) \cdot \nabla u+\mu_{2} u$, i.e., a diffusion-convection-reaction operator with convection field $\boldsymbol{\beta}$. We then introduce a standard Reduced Basis (RB) approximation $[1,4,5]$ for the Crank-Nicolson interpretation of our discrete problem, $u_{N}(\mu) \in$ $\mathcal{X}_{\Delta t, N}=S_{\Delta t} \otimes V_{N}$; here $V_{N} \subset V_{h}$ is an RB space provided for example by the POD-Greedy procedure of [2]. The RB output is then given by $s_{N}=\int_{I} \ell\left(u_{N}(t)\right) d t\left(=\int_{I} \ell\left(\bar{u}_{N}(t)\right) d t\right)$. (It is possible, alternatively, to consider a space-time RB approximation as well [7].) It is then simple [5] to demonstrate

Proposition 5 The $R B$ error satisfies $\left\|\left|u_{\delta}-u_{N}\right|\right\|_{\mathcal{X}, \delta} \leq\left\|r_{N}\right\|_{\mathcal{Y}^{\prime}} / \beta_{\delta}^{\mathrm{LB}}$, where $r_{N}(v):=f(v)-b\left(u_{N}, v\right)$, $\forall v \in \mathcal{Y}$, and $\beta_{\delta}^{L B}$ is a lower bound for the $\beta_{\delta}$ defined in Proposition 3. Furthermore, $\left|s_{\delta}-s_{N}\right| \leq$ $\|\ell\|_{V^{\prime}} \sqrt{T}\left\|r_{N}\right\| \mathcal{Y}^{\prime} / \beta_{\delta}^{L B}$.

The utility of these a posteriori error bounds is critically dependent on the dependence of $\beta_{\delta}$ as a function of the parameter $\mu$ and final time $T, \beta_{\delta}(\mu ; T)$.

We briefly comment on the computational implications of this space-time error bound. As always, our model problem (as is the case here) must honor the "affine-in-functions-of-parameter" condition [5] to permit effective offline-online computation of the reduced basis approximation and a posteriori error bounds. The current formulation introduces the further complication of the space-time norms. In fact, calculation of the dual norm $\|\cdot\|_{\mathcal{Y}^{\prime}}$ (through the corresponding Riesz representation [5]) does not couple different time steps/elements, and hence the additional online difficulty is relatively minor. (The offline stage for computation of the inf-sup lower bound by the Successive Constraint Method [5,8] does require special treatment, in particular due to the norm $\left\|\cdot|\||_{\mathcal{X}, \delta}\right.$.)

\section{Numerical Results}

We report numerical results for the Crank-Nicolson scheme for various choices of the parameters $\mu_{1}, \mu_{2}$ as well as for different time steps $\Delta t$ and uniform mesh sizes $h$. For simplicity, we consider the univariate case (in space) $\Omega=(0,1)$ and choose $\boldsymbol{\beta}(x)=x-\frac{1}{2}$. Let us denote by $\beta_{\delta}(\mu ; T), \gamma_{\delta}(\mu ; T)$ the numerical values for the truth inf-sup and continuity constants, respectively, corresponding to parameter $\mu$ and final time $T$. We start by confirming Proposition 3. Thus, we choose $\mu_{1}=\mu_{2}=0$; for several values of $T, h$, and $\Delta t$ we invariantly obtain 1.000 for both $\beta_{\delta}(\mu ; T)$ and $\gamma_{\delta}(\mu ; T)$, as must be the case.

Next, we investigate the case of convection, $\mu_{2}=0$, in which case $a$ is coercive only for $\mu_{1}<2 \pi^{2}$. We obtain $\beta_{\delta}((50,0) ; 1.0)=0.050, \beta_{\delta}((50,0) ; 2.0)=0.027\left(\gamma_{\delta}=2.60\right)$ and $\beta_{\delta}((100,0) ; 1.0)=0.019$, $\beta_{\delta}((100,0) ; 2.0)=0.010\left(\gamma_{\delta}=5.6\right)$. These numbers are relatively invariant for sufficiently small $h$ and $\Delta t$. We observe numerically an overall behavior of $\beta_{\delta}\left(\left(\mu_{1}, 0\right) ; T\right) \sim\left(\mu_{1} T\right)^{-1}$ and $\gamma_{\delta}\left(\left(\mu_{1}, 0\right) ; T\right) \sim \mu_{1}$ (the latter is readily proven, but not the former). Note $T=\mathcal{O}(1)$ is effectively a "long time" in convective units, $1 / \mu_{1}$. We emphasize that although the problem is non-coercive, the problem is asymptotically stable in the sense that all eigenvalues $\sigma$ of $-a(\psi, \phi)=\sigma\langle\psi, \phi\rangle_{V^{\prime} \times V}$ lie in the left-hand plane; this stability is reflected in the inf-sup behavior. In contrast, a standard energy approach [3] gives effective inf-sup constants on the order of $e^{-\mu_{1} T}$ (here about $10^{-8}$ ). Hence, the traditional method fails to provide useful results, whereas our new approach, which reflects the true time-coupled properties of the system, yields relatively sharp error bounds.

Finally, we consider the case $\mu_{1}=0$ which gives rise to an asymptotically unstable (and non-coercive) system for $\mu_{2}<-\pi^{2}$. This means that any error estimate must grow exponentially with the final time $T$. We observe this for our estimator as well, e.g. $\beta_{\delta}((0,-20) ; 1.0)=3.93 \cdot 10^{-5}\left(\right.$ order of $\left.e^{\mu_{2} T}\right)$. 
Acknowledgements A.T.P. was supported by OSD/AFOSR/MURI Grant FA9550-09-1-0613 and by ONR Grant N00014-11-1-0713; K.U. by the Deutsche Forschungsgemeinschaft (DFG) under Ur-63/9 and GrK1100. The authors are grateful to Sylvain Vallaghé for helpful comments. This paper was written while K.U. was Visiting Professor at MIT.

\section{References}

[1] M. Grepl and A.T. Patera. A posteriori error bounds for reduced-basis approximations of parametrized parabolic partial differential equations. M2AN Math. Model. Numer. Anal. 39 (2005), no. 1, 157-181.

[2] B. Haasdonk and M. Ohlberger. Reduced basis method for finite volume approximations of parametrized linear evolution equations. M2AN Math. Model. Numer. Anal. 42 (2008), 277-302.

[3] D.J. Knezevic, N.C. Nguyen, and A.T. Patera. Reduced basis approximation and a posteriori error estimation for the parametrized unsteady Boussinesq equations. Math. Mod. Meth. Appl. Sci., 21 no. 7 (2011), 1415-1442.

[4] D.V. Rovas, L. Machiels, and Y. Maday. Reduced-basis output bound methods for parabolic problems. IMA J. Numer. Anal. 26 no. 3 (2006), 423-445.

[5] G. Rozza, D.B.P. Huynh, and A.T. Patera. Reduced basis approximation and a posteriori error estimation for affinely parametrized elliptic coercive partial differential equations - Application to transport and continuum mechanics. Arch. Comp. Meth. Eng., 15 no. 3 (2008), 229-275.

[6] C. Schwab and R. Stevenson. Space-time adaptive wavelet methods for parabolic evolution problems. Math. Comp. 78 (2009), 1293-1318.

[7] K. Steih and K. Urban. Space-time reduced basis methods for time-periodic parabolic problems. University of Ulm, Preprint 2012. www.uni-ulm.de/mawi/fakultaet/forschung/preprint-server.html

[8] S. Vallaghé, A. Le-Hyaric, M. Fouquemberg, and C. Prud'homme. A successive constraint method with minimal offline constraints for lower bounds of parametric coercivity constant. Preprint: hal-00609212, hal.archives-ouvertes.fr. 